\title{
Eñes
}

\section{Investigation of the $\mathrm{SnS} / \mathrm{Cu}_{2} \mathrm{ZnSnS}_{4}$ Interfaces in Kesterite Thin-Film Solar Cells}

\author{
Yi Ren, ${ }^{*}{ }^{\S}$ Michael Richter, ${ }^{\dagger}$ Jan Keller, ${ }^{\S}$ Alex Redinger, ${ }^{\ddagger}$ Thomas Unold, ${ }^{\ddagger}$ Olivier Donzel-Gargand, ${ }^{\S}$
} Jonathan J. S. Scragg, and Charlotte Platzer Björkman ${ }^{\S}$

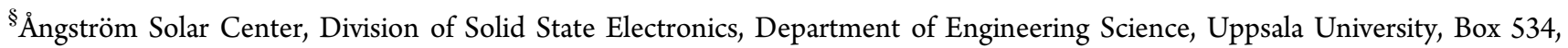
SE-75121 Uppsala, Sweden

${ }^{\dagger}$ Laboratory for Chalcogenide Photovoltaics, Energy and Semiconductor Research Laboratory, University of Oldenburg, 26111 Oldenburg, Germany

${ }^{\ddagger}$ Helmholtz-Zentrum Berlin für Materialien und Energie (HZB), Hahn-Meitner-Platz 1, D-14109 Berlin, Germany

\section{Supporting Information}

ABSTRACT: Kesterite $\mathrm{Cu}_{2} \mathrm{ZnSnS}_{4}$ (CZTS), having only earthabundant elements, is a promising solar cell material. Nevertheless, the impact of the SnS secondary phase, which often forms alongside CZTS synthesis at high annealing temperature, on CZTS solar cells is poorly studied. We confirm, by means of X-ray diffraction, Raman scattering, and energy dispersive $\mathrm{X}$-ray spectroscopy mapping, that this phase tends to segregate at both the surface and the back side of annealed CZTS films with $\mathrm{Cu}$-poor and $\mathrm{Zn}$-rich composition. Using electron beam-induced current measurements, it is further demonstrated that the formation of SnS on the CZTS surface is harmful for solar cells, whereas the

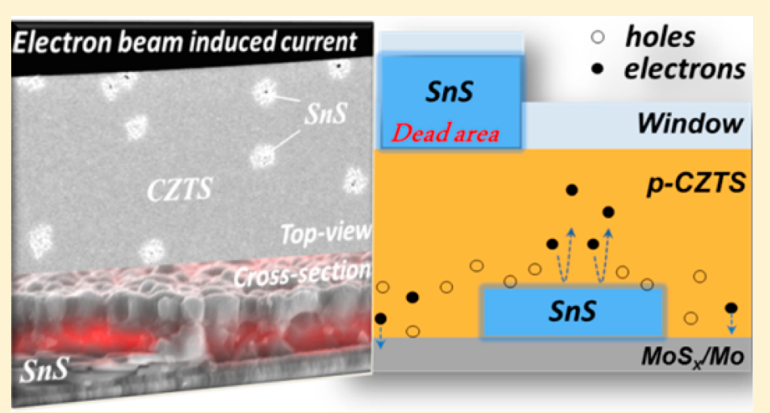
SnS phase can be beneficial for solar cells when it segregates on the CZTS rear. This positive contribution of SnS could stem from a passivation effect at the CZTS/SnS rear interface. This work opens new possibilities for an alternative interface development for kesterite-based photovoltaic technology.

$\mathrm{T}$ o attain low material cost for solar cell technology, kesterite $\mathrm{Cu}_{2} \mathrm{ZnSnS}_{4}$ (CZTS)-based material has attracted tremendous attention in the past years because of its all earth-abundant elements. To date, the record efficiency of CZTS solar cells has reached 9.5\%. ${ }^{1}$ Nevertheless, further enhancement of this technology is required in order to target above $15 \%$ efficiency level for industrial implementation. One main issue that inhibits the improvement of CZTS solar cells is the understanding of the influence from the secondary phases. Since most of the CZTS solar cells with highest efficiency have $\mathrm{Zn}$-rich composition, ${ }^{2}$ and since it was suggested that $\mathrm{Sn}$-rich composition can limit the surface decomposition of CZTS at high annealing temperature, ${ }^{3}$ the current CZTS film is usually prepared under $\mathrm{Cu}$-poor and $\mathrm{Zn}$-rich composition. This leads to formation of $\mathrm{ZnS}$ and $\mathrm{SnS}$ secondary phases being most commonly seen and reported in the literature. ${ }^{4-6}$ The effect of ZnS segregated at CZTS interfaces on solar cells has already been intensively studied. For instance, by intentionally controlling $\mathrm{Zn}$ flux via thermal evaporation to segregate $\mathrm{ZnSe}$ phases, Hsu et al. found that $\mathrm{ZnSe}$ was harmless if situated on the back side of CZTSe but detrimental on the surface of $\mathrm{Cu}_{2} \mathrm{ZnSnSe}_{4}{ }^{7}$ In contrast, studies on the impact of $\mathrm{SnS}$ phases on CZTS solar cells are scarce. A wet etching process was developed by Xie et al., and removal of $\operatorname{Sn}(\mathrm{S}, \mathrm{Se})$ phases on the surface of $\mathrm{Cu}_{2} \mathrm{ZnSn}(\mathrm{S}, \mathrm{Se})_{4}$ films was shown to improve solar cell performance. ${ }^{8}$ Because the etchant solution can simultaneously react with the CZTSSe surface when etching $\mathrm{Sn}(\mathrm{S}, \mathrm{Se})$, these effects are difficult to separate.

The aim of this Letter is to explore the impact of $\mathrm{SnS}$ phases on CZTS solar cells. We exploited electron beam-induced current (EBIC) measurements to investigate the role of the SnS phase in CZTS solar cells. The merit of this approach is to provide a direct comparison of the local electrical property of the solar cell with and without the appearance of $\mathrm{SnS}$. To complement this electrical measurement and to achieve a better correlation, we also performed material characterization to determine the locations of SnS in CZTS films. The results demonstrate that SnS segregation on the back side of CZTS films can potentially increase the short-circuit current $\left(J_{\mathrm{sc}}\right)$ of the CZTS solar cells.

The CZTS films investigated in this work were prepared from reactive cosputtering of $\mathrm{CuS}, \mathrm{Zn}$, and $\mathrm{Sn}$ targets, to a uniform thickness of $700 \mathrm{~nm}$ and a composition of $\mathrm{Cu} / \mathrm{Sn}=1.8$ and $\mathrm{Zn} / \mathrm{Sn}=1.1$. The as-sputtered film was then annealed at

Received: February 23, 2017

Accepted: April 6, 2017 
(a)

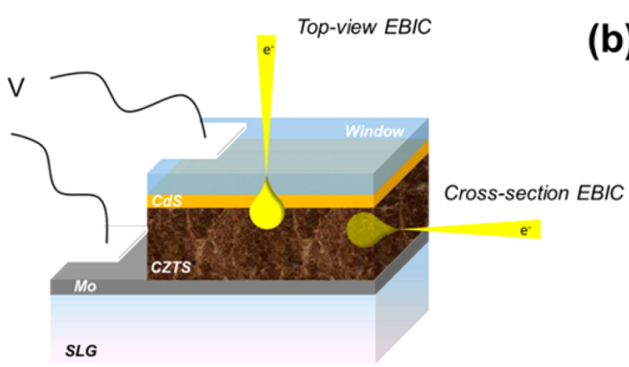

(b)

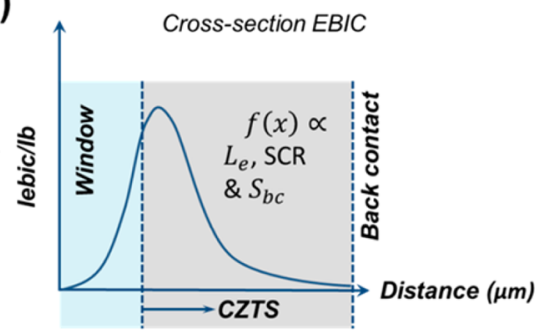

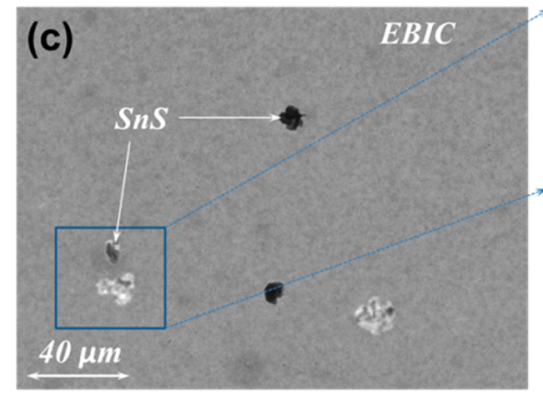

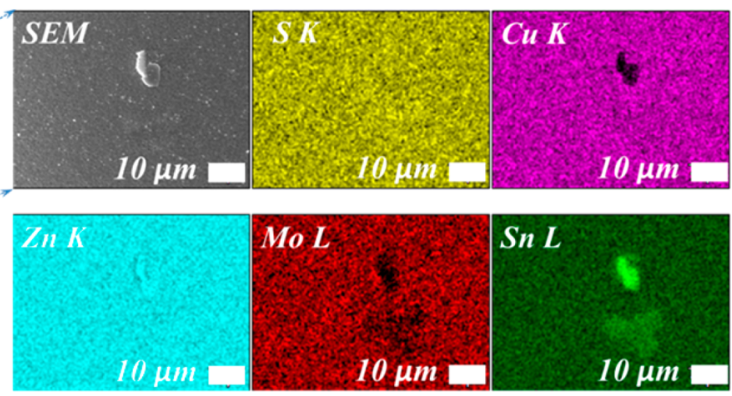

Figure 1. (a) Electron beam induced current (EBIC) setup with two configurations. (b) Cross-sectional EBIC current profile that is dependent on the collection function $f(x)$ [influenced by carrier diffusion length $\left(L_{\mathrm{e}}\right)$, space charge region width (SCR), and back contact recombination velocity $\left(S_{\mathrm{bc}}\right)$ ]. (c) Top-view EBIC and energy-dispersive X-ray spectroscopy (EDS) mapping on the CZTS solar cell device. A bright EBIC signal is clearly visible in the blue square area, but there is no obvious feature in secondary electron view, which indicates that it is located below the surface. EDS mapping (performed at $20 \mathrm{keV}$ ) on the same area shows a higher signal of Sn only.

about $560{ }^{\circ} \mathrm{C}$ for $10 \mathrm{~min}$ under $350 \mathrm{mbar}$ static Ar within sulfur excess environment. A standard solar cell structure of Al-doped $\mathrm{ZnO}(210 \mathrm{~nm}) / \mathrm{i}-\mathrm{ZnO}(80 \mathrm{~nm}) / \mathrm{n}$-type $\mathrm{CdS}(60 \mathrm{~nm})$ was used including $\mathrm{KCN}$ etching of the absorber after annealing. More details can be found elsewhere. ${ }^{9}$ Lastly, $\mathrm{Ni} / \mathrm{Al} / \mathrm{Ni}$ front contact was evaporated on top of the device for electrical characterization and EBIC measurements. The mechanically scribed solar cell device of $0.5 \mathrm{~cm}^{2}$ had an efficiency of $3.9 \%$.

Figure 1c presents the EBIC measurement from the top-view configuration as indicated in Figure 1a. When this is compared to the CZTS matrix in the EBIC image, additional darker and brighter areas can be noticed. To determine the origin of these features, EDS mapping was recorded on the area indicated by the blue rectangle in Figure 1c. It can be confirmed that the area with poorer (dark) EBIC signal is attributed to a Sn-rich and $\mathrm{Zn}$-rich phase, while the area with stronger (bright) EBIC signal results from a $\mathrm{Sn}$-rich phase. A further resolution of $\mathrm{S} \mathrm{K}$ and Mo $\mathrm{L}$ energies allows us to deduce that the EBIC features come from the $\mathrm{SnS}$ phases, since the distribution of sulfur is homogeneous over the examined area. It is not unexpected to observe a uniform $\mathrm{Zn}$ signal spanning the entire sample because the solar cell stack is terminated with a $\mathrm{ZnO}$ layer.

To verify the formation of $\mathrm{SnS}$, we investigated the bare CZTS absorber after annealing. Figure $2 \mathrm{a}$ shows the comparison of X-ray diffraction (XRD) patterns of the annealed CZTS films, with Bragg-Brentano and grazing incidence (GIXRD) configurations. As indicated by the small intensity of the Mo peak in Figure 2a, the angle of GIXRD allowed the measurement of the entire CZTS film. It is noticeable in Bragg-Brentano XRD that besides the Mo and CZTS phases, the orthorhombic $\mathrm{SnS}$ phase can be detected, with the peaks at $31.9^{\circ}(004)$ and $66.7^{\circ}(008)$. These two peaks vanished in GIXRD, suggesting that the SnS phase might be textured. To locate SnS in the CZTS film, Raman scattering was performed on both the surface and back side of the CZTS film. To preserve the features at the CZTS rear, we glued the CZTS film to another glass substrate and mechanically lifted it off at the interface of $\mathrm{CZTS} / \mathrm{MoS}_{x}$. The inset of Figure $2 \mathrm{~b}$ displays an apparent phase contrast from optical microscopy on the $\mathrm{MoS}_{x} /$ Mo substrate after CZTS removal. Raman scattering (Figure 2b) shows that the bright zones in the optical image, which coincide with places with enhanced and reduced EBIC signals in Figure 1c, are attributed to $\mathrm{SnS}$ (main vibrational mode at 187,157 , and $221 \mathrm{~cm}^{-1}$ ). The surrounding (Figure $2 \mathrm{~b}$, orange curve) is clearly identified as $\mathrm{MoS}_{x}$. The shape of $\mathrm{SnS}$ is a dendrite structure rather than a complete platelike appearance. By correlating the coverage of brighter areas in Figure 1c and Figure $2 \mathrm{~b}$, we find that the calculated coverage percentage of about $1-2 \%$ matches very well (Figure S2), implying that SnS on the back side of CZTS films could be the reason for the increased EBIC signal. Figure $2 c$ shows the cross-sectional EDS mapping of the CZTS absorber, revealing that the thickness of the segregated SnS phases on the CZTS rear is $200 \pm 50 \mathrm{~nm}$, while those on the CZTS surface are above $1 \mu \mathrm{m}$ (Figure S3). The SnS phase on the CZTS rear could cause a lateral variation of the effective CZTS thickness, as we observed that the thickness of the CZTS layer on top of $\mathrm{SnS}$ could change from 450 to $700 \mathrm{~nm}$ depending on the positions for SEM images (see Figures S3, S4, and 2c). Furthermore, we also noticed in Figure $2 c$ that $\mathrm{SnS}$ has a densely packed layer structure, which agrees with the analysis of the XRD pattern in Figure 2a that the orthorhombic SnS formed in the annealed CZTS film likely has a preferential orientation along the [001] direction. According to EDS mapping on the CZTS cross section, we cannot find an apparent SnS phase formed in the bulk CZTS. Therefore, within the limitation of characterization techniques, the combined results from EDS mapping, Raman scattering, and XRD measurements lead us to conclude that SnS locates only at the interfaces in the CZTS solar cell.

It is interesting to note from the EBIC measurements how the influence of $\mathrm{SnS}$ on CZTS solar cells is connected to its position. As indicated by the dark area in Figure 1c, the thick SnS phases formed on the CZTS surface behave as dead areas for the solar cell, because no EBIC current can be extracted 
(a)

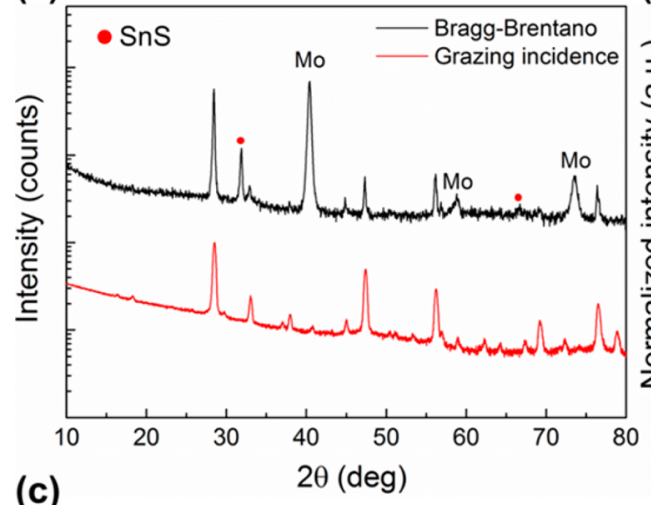

(b)

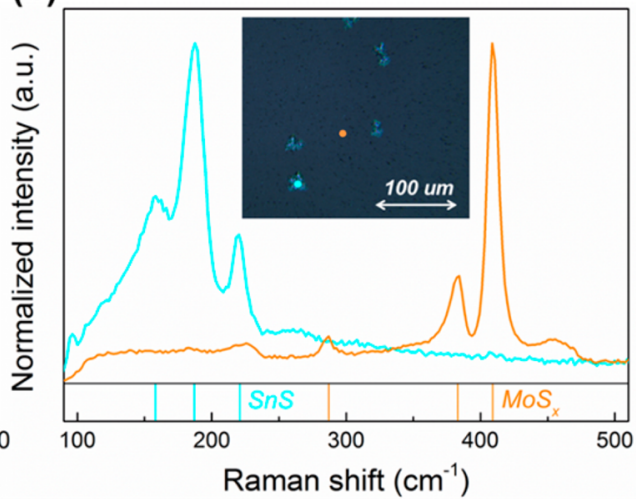

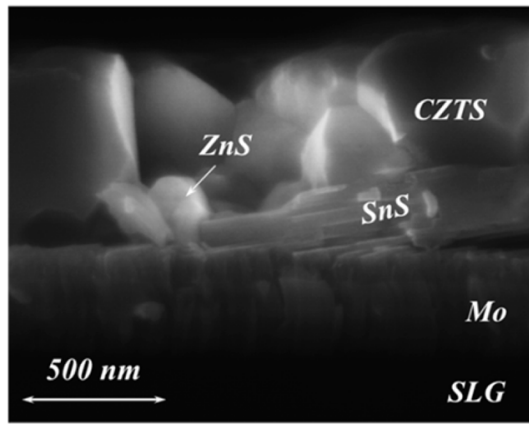
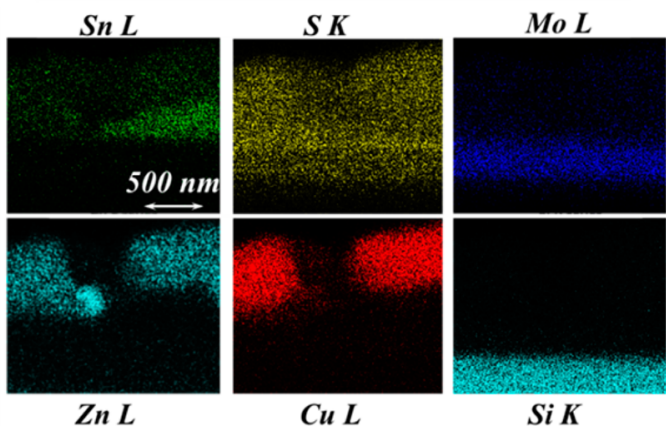

Figure 2. (a) Comparison of the X-ray diffraction pattern with two configurations; (b) Raman scattering (532 $\mathrm{nm}$ laser wavelength) on the $\mathrm{MoS}_{x} / \mathrm{Mo} / \mathrm{SLG}$ substrate from which the annealed CZTS film was mechanically lifted off (optical image in the inset). The dots in the optical image refer to the measured spots and correspond to the Raman spectrum with the same color. (c) EDS mapping at $7 \mathrm{keV}$ on the SEM cross section of the annealed CZTS film.

from these places. SnS is a p-type solar absorber material; therefore, it is expected that this phase can respond to the EBIC measurement, as was reported recently. ${ }^{10}$ The reasons for the results in Figure 1c could be that either the collection of charge carriers in the SnS phase is extremely poor, because of a missing electrical field and/or a very limited electron diffusion length in $\mathrm{SnS}$, or that the $\mathrm{SnS}$ phase was poorly integrated in the solar cell (e.g., edge of SnS or SnS/CZTS contact). The dead areas induced by the $\mathrm{SnS}$ phase on CZTS surface agree with the observation that the $J_{\mathrm{sc}}$ of the solar cell can be improved when these phases were removed by $\left(\mathrm{NH}_{4}\right)_{2} \mathrm{~S}$ etching. ${ }^{8}$ Nevertheless, $\mathrm{SnS}$ has the potential to be beneficial for the $J_{\mathrm{sc}}$ of the solar cell when it is formed on the CZTS rear. To understand this behavior, we repeated the experiment by preparing a CZTS film of about $650 \mathrm{~nm}$ with composition similar to that of the samples studied in Figure 1. The respective EBIC measurements on the final solar cells are shown in Figure 3a. It is important to note that the effect of the $\mathrm{SnS}$ phase on CZTS solar cell is repeatable. It can be found in Figure $3 \mathrm{~b}$ that the EBIC signal from the brighter zones is about $30 \%$ higher than that from the CZTS matrix, which gives an indication of the level of $J_{\text {sc }}$ enhancement of the solar cell that could possibly be obtained.

To gain deeper insight behind the positive influence of SnS on CZTS rear, cross-sectional EBIC measurement of the CZTS solar cell was conducted. At first sight, we notice several characteristics from the overlay image of SEM and EBIC in Figure 3c: (i) SnS on the surface and back side of the CZTS film contributes no EBIC current, and (ii) the EBIC signal seems stronger on the CZTS film that is above SnS than the film that is directly above $\operatorname{MoS}_{x}$. (See more EBIC images in Figure S5.) Figure 3d illustrates the normalized EBIC profiles that are extracted from the marked areas in Figure 3c. It can be noted that the EBIC signal becomes stronger in CZTS when $\mathrm{SnS}$ is present beneath CZTS. This is consistent with the observation from top-view EBIC in Figure $3 \mathrm{~b}$, demonstrating that the CZTS film on top of $\mathrm{SnS}$ has better charge carrier collection. In addition, one can note that there is a peak toward the end of all EBIC profiles, which originates from the tilted sample stage. However, the artificial peak could indicate that the back contact was reached, i.e., either $\operatorname{SnS}$ or $\mathrm{MoS}_{x}$, which we will use as a boundary for the EBIC analysis. As already indicated in Figure $1 b$, the cross-sectional EBIC profile can be treated as a one-dimensional convolution of the lateral generation profile $g(x, \mathrm{~A})$ at the irradiance position $\mathrm{A}$ and the collection function $f(x)$ in the $x$ direction perpendicular to the normal of the cross-sectional surface. Therefore, we can analytically simulate the EBIC profile to match with the experimental data for further analysis. The details of EBIC simulation are explained in the Supporting Information.

Figure $3 e$ presents the analytically simulated EBIC profiles corresponding to the data in Figure $3 \mathrm{~d}$ (the color represents the respective positions in Figure $3 c, d)$. The fitted electron diffusion length in CZTS $\left(L_{\mathrm{e}}\right)$ and the space charge region width (SCR) are in good agreement with our previous thickness study of the CZTS films with comparable composition and literature reports. ${ }^{9,11,12}$ Furthermore, we find that the back contact recombination velocity $\left(S_{\mathrm{bc}}\right)$ for position 2 is about 3 orders of magnitude lower than that for position 3 . Note that the thickness of the CZTS film assumed for position 2 is thinner $(450 \mathrm{~nm})$ than that of position $3(600 \mathrm{~nm})$ based on the consideration of the CZTS thickness variation in Figure 2c. The EBIC simulation suggests that $\mathrm{SnS}$ improves the charge carrier collection by means of reducing $S_{\mathrm{bc}}$. Because of the 

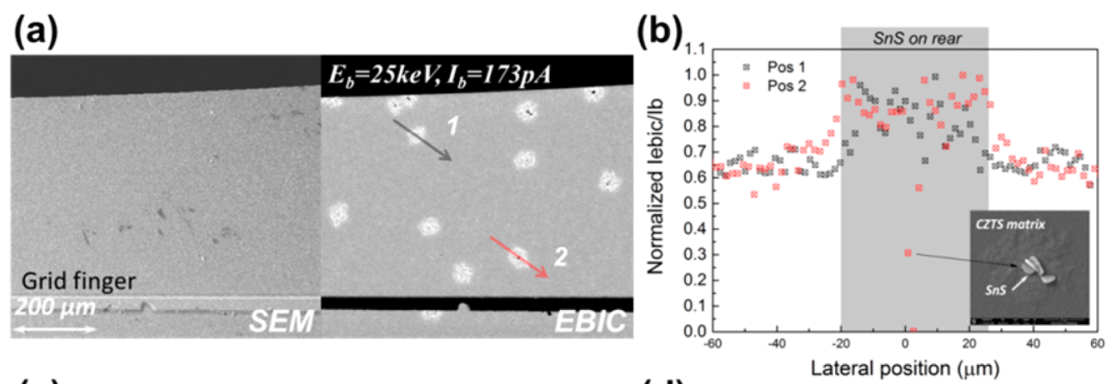

(c)

(d)
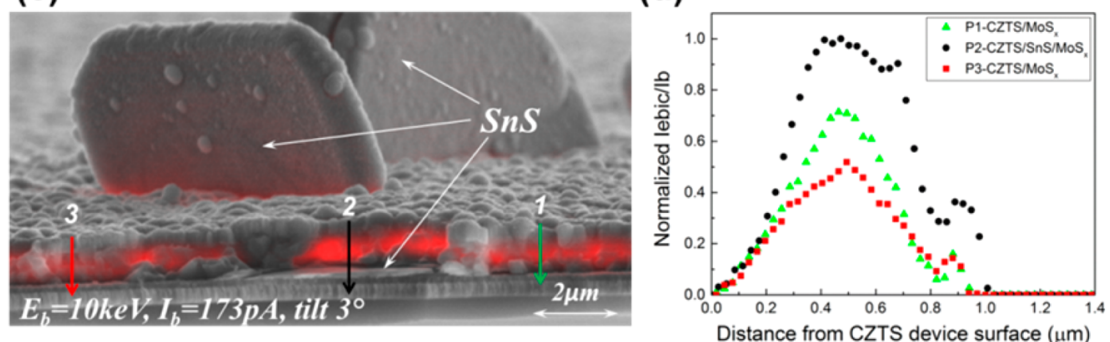

(e)

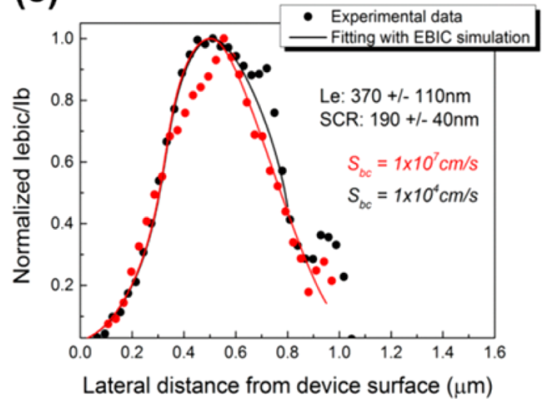

(f)

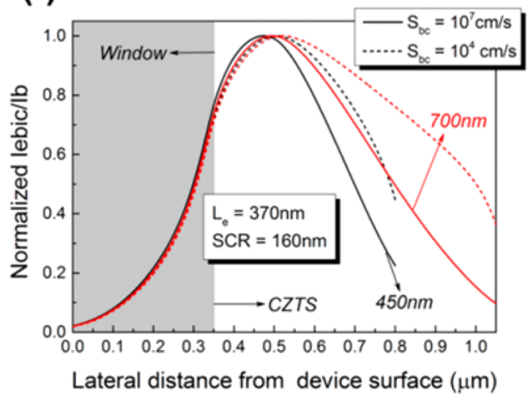

Figure 3. (a) Top-view EBIC and SEM images and the extracted EBIC current profiles based on the line scans shown in panel b. The arrow of the line scan in the EBIC image refers to the scan direction. The inset in panel b shows SnS on the CZTS surface, which displays no EBIC current. (c) Overlay cross-sectional images of SEM and EBIC (red). The arrow of the line scan refers to the scan direction. (d) Extracted EBIC profiles based on the respective line scans that are shown in panel c. (e) Analytical simulation of the cross-sectional EBIC profile to fit the experimental data shown in panel d with different $S_{\mathrm{bc}}$ (f) Comparison of the analytical EBIC simulations with different $S_{\mathrm{bc}}$ and CZTS thickness.
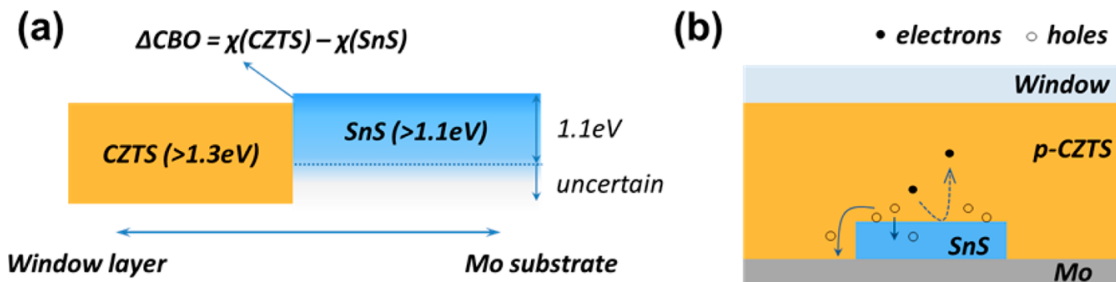

Figure 4. Proposed (a) band edges at the interface of CZTS/SnS and (b) charge carrier transport paths close to CZTS/SnS interface.

necessity of varying CZTS thickness for the EBIC fitting in Figure $3 \mathrm{e}$, it is important to take a closer look at the effect of CZTS thickness in correlation to the $S_{\mathrm{bc}}$ on EBIC simulation. In Figure $3 \mathrm{f}$, it is demonstrated that a decrease in $S_{\mathrm{bc}}$, in particular with the given $L_{\mathrm{e}}$ and SCR in Figure 3e, affects the charge carrier collection. On one hand, this implies that if the CZTS thickness is altered due to $\mathrm{SnS}$, it is possible that the EBIC profile shows limited difference (see dashed black and solid red curves in Figure 3f). Nonetheless, when the effective CZTS thickness is uniform regardless of $\mathrm{SnS}$ formation at the CZTS rear, the number of collected charge carriers should deviate significantly because the effect of $S_{\mathrm{bc}}$ will dominate [see solid and dashed black (red) curves in Figure 3f], which should result in evident contrast in the EBIC signal. The above discussion could explain the variation of the EBIC contrast in
Figure $3 \mathrm{~b}$. Moreover, we find that after cross-sectional EBIC profiles in Figure $3 \mathrm{f}$ are integrated, the total EBIC current for small $S_{\mathrm{bc}}$ (dased line) is about $10 \%$ and $20 \%$ higher than for high $S_{\mathrm{bc}}$ (solid line) for 450 and $700 \mathrm{~nm}$ thick CZTS, respectively. This presumed increment is comparable to the top-view EBIC result in Figure 3b, which shows 30\% enhancement of EBIC signal on areas with $\mathrm{SnS}$ appearing at CZTS rear. The detailed and integral EBIC analysis strongly suggests that $\mathrm{SnS}$ improves the charge carrier collection in CZTS possibly owing to reduction in $S_{\mathrm{bc}}$ at the CZTS/SnS rear interface. Nevertheless, another possibility, which is difficult to confidently separate from reduction in $S_{\mathrm{bc}}$ could be that the presence of SnS may alter the bulk defect properties of CZTS in the vicinity of the SnS phase giving a change in $L_{\mathrm{e}}$, as indicated by the error of the fitted result in Figure $3 \mathrm{e}$. 
It is interesting now to understand how the $\mathrm{SnS}$ phase on the CZTS rear can be beneficial for the solar cell. Considering the reported indirect optical band gap of about $1.1 \mathrm{eV}$ for p-type $\mathrm{SnS}$ and the narrowed band gap of $1.3 \mathrm{eV}$ for p-type CZTS (e.g., resulting from potential fluctuations) ${ }^{13,14}$ we can sketch the isolated band structure of the possible $\mathrm{p}-\mathrm{p}$ heterojunction at the CZTS/SnS interface in Figure 4a. The anticipated spikelike conduction band offset $(\Delta \mathrm{CBO}$, typically $>0 \mathrm{eV})$ at this interface is in accordance with theoretical calculations and comparisons of the CBO offsets for CdS/CZTS and CdS/ $\mathrm{SnS}^{15-17}$ If CZTS connects to $\mathrm{SnS}$ as seen in Figure 4a, the spikelike $\mathrm{CBO}$ would limit electron transport toward the back contact, giving a passivation effect. For this, a comparable valence band maximum of $\mathrm{SnS}$ to CZTS is also required to equalize the Fermi level at the thermal equilibrium. In fact, the true band edge of orthorhombic SnS, particularly when it is integrated to solar cell devices, remains under debate because the band gap derived from quantum efficiency of $\mathrm{SnS}$ solar cells is usually about $1.3 \mathrm{eV}^{18-20}$ which is higher than the commonly reported indirect optical band gap of $1.1 \mathrm{eV}$. $^{14}$ Another possibility for the band edge variation could be structural disorder, which includes the influence from either the defect constitution (such as defect pairs in $\mathrm{SnS}$, analogous to CZTS 2), or a mixture of SnS polymorph like cubic SnS phase (band gap of above $1.5 \mathrm{eV}^{21,22}$ ) within a nanoscale size that is enough to shift the band edge close to the interface. As a result, the CZTS/SnS interface could behave as a mirror to reflect the electrons back toward the front contact of the CZTS solar cell, as drawn in Figure $4 b$.

One important aspect that we cannot discard is that the real operation of a solar cell is different from the EBIC measurement because of the difference in the generation of electron-hole pairs. The generation of charge carriers during operation of a solar cell depends on the optical absorption. In fact, our preliminary optical simulation (not shown here) also reveals that when $\mathrm{SnS}$ is present on the CZTS rear, the back surface reflection of the near-infrared light could be enhanced, leading to a slight increase of the light absorption in CZTS. Such results further indicate that $\mathrm{SnS}$ might be useful in facilitating light management of the solar cell. Nevertheless, it is still important to improve and control the quality of the bulk CZTS. The spontaneously segregated SnS phase from CZTS film could reversely alter the local composition of CZTS, causing lateral structural inhomogeneities. The net effect of the influences from the poor CZTS bulk and from the beneficial CZTS/SnS rear interface can still result in overall deterioration of the solar cell performance.

The demonstrated and discussed CZTS/SnS interface properties indicate that a careful control of the grown $\mathrm{SnS}$ phase is crucial. It is notable via EBIC measurement that the spontaneously formed $\mathrm{SnS}$ on the CZTS surface should be eliminated, while $\mathrm{SnS}$ should be controlled on the CZTS rear. Formation of $\mathrm{SnS}$ on the CZTS surface could stem from the process conditions ${ }^{8}$ and CZTS composition. ${ }^{3}$ It has been shown that both etching with $\left(\mathrm{NH}_{4}\right)_{2} \mathrm{~S}$ solution and using thermal evaporation during the annealing process are effective approaches for removing SnS on CZTS surface. ${ }^{8}$ Regarding the CZTS rear, SnS could form due to the initial CZTS composition $^{23}$ as well as the back contact decomposition. ${ }^{24}$ Therefore, to control the SnS formation on the CZTS rear, efforts to develop suitable CZTS composition and to passivate the back contact are required. Nonetheless, to better underline the passivation effect of $\mathrm{SnS}$ on CZTS and to further improve the interface properties in CZTS-based technology in general, it is a prerequisite to provide a solid basis of high-quality CZTS phase.

In summary, the influence of the $\mathrm{SnS}$ secondary phase on CZTS solar cells is investigated via EBIC measurements. It is found that the $\mathrm{SnS}$ phase appears only on the surface and back side of CZTS films. SnS phases on the CZTS surface introduce dead areas to the solar cell, whereas $\mathrm{SnS}$ can be beneficial for the $J_{\text {sc }}$ of the solar cell when it appears on the CZTS rear. This contribution of $\mathrm{SnS}$ could result from a passivation effect at the CZTS/SnS back side interface. Our findings are of importance to the potential interface development for thin-film photovoltaics with earth-abundant material.

\section{ASSOCIATED CONTENT}

\section{S Supporting Information}

The Supporting Information is available free of charge on the ACS Publications website at DOI: 10.1021/acsenergylett.7b00151.

Methods including the details of the material characterizations and analytical EBIC simulation, as well as additional SEM and EBIC images for statistics (PDF)

\section{AUTHOR INFORMATION}

\section{Corresponding Author}

*E-mail: yi.ren@angstrom.uu.se. ORCID

Olivier Donzel-Gargand: 0000-0002-2101-3746

Notes

The authors declare no competing financial interest.

\section{ACKNOWLEDGMENTS}

The financial support leading to this research is kindly acknowledged, including the People Program (Marie Curie Actions) of the European Union's seventh Framework Program FP7/2007-2013/under REA Grant Agreement No. 316488 (KESTCELLS), and partly by the Knut \& Alice Wallenberg Foundation, Swedish Foundation for Strategic Research, and Swedish Science Foundation. A.R. thanks the Fonds national de la recherche Project 7842175 for funding. Y.R. thanks Dr. ShuYi Li, Dr. Oleksandr Bilousov, and Dr. Carl Hägglund for fruitful discussion of optical simulation and material properties of $\mathrm{SnS}$.

\section{REFERENCES}

(1) Green, M. A.; Emery, K.; Hishikawa, Y.; Warta, W.; Dunlop, E. D.; Levi, D. H.; Ho-Baillie, A. W. Y. Solar Cell Efficiency Tables (Version 49). Prog. Photovoltaics 2016, 24, 3-13.

(2) Chen, S.; Walsh, A.; Gong, X.-G.; Wei, S.-H. Classification of Lattice Defects in the Kesterite $\mathrm{Cu}_{2} \mathrm{ZnSnS}_{4}$ and $\mathrm{Cu}_{2} \mathrm{ZnSnSe}_{4}$ EarthAbundant Solar Cell Absorbers. Adv. Mater. 2013, 25, 1522-1539.

(3) Scragg, J. J.; Ericson, T.; Kubart, T.; Edoff, M.; Platzer-Björkman, C. Chemical Insights into the Instability of $\mathrm{Cu}_{2} \mathrm{ZnSnS}_{4}$ Films During Annealing. Chem. Mater. 2011, 23, 4625-4633.

(4) Xie, H.; Dimitrievska, M.; Fontané, X.; Sánchez, Y.; LópezMarino, S.; Izquierdo-Roca, V.; Bermúdez, V.; Pérez-Rodríguez, A.; Saucedo, E. Formation and Impact of Secondary Phases in Cu-poor $\mathrm{Zn}$-rich $\mathrm{Cu}_{2} \mathrm{ZnSn}\left(\mathrm{S}_{1-\mathrm{y}} \mathrm{Se}_{\mathrm{y}}\right)_{4}(0 \leq y \leq 1)$ Based Solar Cells. Sol. Energy Mater. Sol. Cells 2015, 140, 289-298.

(5) Li, W.; Chen, J.; Yan, C.; Hao, X. The Effect of ZnS Segregation on Zn-rich CZTS Thin Film Solar Cells. J. Alloys Compd. 2015, 632, 178-184. 
(6) Feng, Y.; Lau, T.-K.; Cheng, G.; Yin, L.; Li, Z.; Luo, H.; Liu, Z.; Lu, X.; Yang, C.; Xiao, X. A Low-Temperature Formation Path Toward Highly Efficient Se-Free $\mathrm{Cu}_{2} \mathrm{ZnSnS}_{4}$ Solar Cells Fabricated Through Sputtering and Sulfurization. CrystEngComm 2016, 18, 1070-1077.

(7) Hsu, W.-C.; Repins, I.; Beall, C.; DeHart, C.; Teeter, G.; To, B.; Yang, Y.; Noufi, R. The Effect of Zn Excess on Kesterite Solar Cells. Sol. Energy Mater. Sol. Cells 2013, 113, 160-164.

(8) Xie, H.; Sanchez, Y.; Lopez-Marino, S.; Espindola-Rodriguez, M.; Neuschitzer, M.; Sylla, D.; Fairbrother, A.; Izquierdo-Roca, V.; PerezRodriguez, A.; Saucedo, E. Impact of $\mathrm{Sn}(\mathrm{S}, \mathrm{Se})$ Secondary Phases in $\mathrm{Cu}_{2} \mathrm{ZnSn}(\mathrm{S}, \mathrm{Se})_{4}$ Solar Cells: a Chemical Route for Their Selective Removal and Absorber Surface Passivation. ACS Appl. Mater. Interfaces 2014, 6, 12744-51.

(9) Ren, Y.; Scragg, J. J. S.; Frisk, C.; Larsen, J. K.; Li, S.-Y.; PlatzerBjörkman, C. Influence of the $\mathrm{Cu}_{2} \mathrm{ZnSnS}_{4}$ Absorber Thickness on Thin Film Solar Cells. Phys. Status Solidi A 2015, 212, 2889-2896.

(10) Steinmann, V.; Chakraborty, R.; Rekemeyer, P. H.; Hartman, K.; Brandt, R. E.; Polizzotti, A.; Yang, C.; Moriarty, T.; Gradečak, S.; Gordon, R. G.; Buonassisi, T. A Two-Step Absorber Deposition Approach To Overcome Shunt Losses in Thin-Film Solar Cells: Using Tin Sulfide as a Proof-of-Concept Material System. ACS Appl. Mater. Interfaces 2016, 8, 22664-22670.

(11) Just, J.; Nichterwitz, M.; Lützenkirchen-Hecht, D.; Frahm, R.; Unold, T. Compositional Dependence of Charge Carrier Transport in Kesterite $\mathrm{Cu}_{2} \mathrm{ZnSnS}_{4}$ Solar Cells. J. Appl. Phys. 2016, 120, 225703.

(12) Pu, A.; Ma, F.; Yan, C.; Huang, J.; Sun, K.; Green, M.; Hao, X. Sentaurus Modelling of $6.9 \% \mathrm{Cu}_{2} \mathrm{ZnSnS}_{4}$ Device Based on Comprehensive Electrical \& Optical Characterization. Sol. Energy Mater. Sol. Cells 2017, 160, 372-381.

(13) Zawadzki, P.; Zakutayev, A.; Lany, S. Entropy-Driven Clustering in Tetrahedrally Bonded Multinary Materials. Phys. Rev. Appl. 2015, 3, 034007.

(14) Banai, R. E.; Horn, M. W.; Brownson, J. R. S. A Review of Tin (II) Monosulfide and its Potential as a Photovoltaic Absorber. Sol. Energy Mater. Sol. Cells 2016, 150, 112-129.

(15) Abdel Haleem, A. M.; Ichimura, M. Experimental Determination of Band Offsets at the $\mathrm{SnS} / \mathrm{CdS}$ and $\mathrm{SnS} / \mathrm{InS}_{\mathrm{x}} \mathrm{O}_{\mathrm{y}}$ Heterojunctions. J. Appl. Phys. 2010, 107, 034507.

(16) Bär, M.; Schubert, B. A.; Marsen, B.; Wilks, R. G.; Pookpanratana, S.; Blum, M.; Krause, S.; Unold, T.; Yang, W.; Weinhardt, L.; Heske, C.; Schock, H. W. Cliff-Like Conduction Band Offset and KCN-Induced Recombination Barrier Enhancement at the $\mathrm{CdS} / \mathrm{Cu}_{2} \mathrm{ZnSnS}_{4}$ Thin-Film Solar Cell Heterojunction. Appl. Phys. Lett. 2011, 99, 222105.

(17) Burton, L. A.; Walsh, A. Band Alignment in SnS Thin-Film Solar Cells: Possible Origin of the Low Conversion Efficiency. Appl. Phys. Lett. 2013, 102, 132111.

(18) Schneikart, A.; Schimper, H. J.; Klein, A.; Jaegermann, W. Efficiency Limitations of Thermally Evaporated Thin-Film SnS Solar Cells. J. Phys. D: Appl. Phys. 2013, 46, 305109.

(19) Ikuno, T.; Suzuki, R.; Kitazumi, K.; Takahashi, N.; Kato, N.; Higuchi, K. SnS Thin Film Solar Cells with $\mathrm{Zn}_{1-\mathrm{x}} \mathrm{Mg}_{\mathrm{x}} \mathrm{O}$ Buffer Layers. Appl. Phys. Lett. 2013, 102, 193901.

(20) Ramakrishna Reddy, K. T.; Koteswara Reddy, N.; Miles, R. W. Photovoltaic Properties of SnS Based Solar Cells. Sol. Energy Mater. Sol. Cells 2006, 90, 3041-3046.

(21) Abutbul, R. E.; Segev, E.; Zeiri, L.; Ezersky, V.; Makov, G.; Golan, Y. Synthesis and Properties of Nanocrystalline $\pi$-SnS - a New Cubic Phase of Tin Sulphide. RSC Adv. 2016, 6, 5848-5855.

(22) Bilousov, O. V.; Ren, Y.; Törndahl, T.; Donzel-Gargand, O.; Ericson, T.; Platzer-Björkman, C.; Edoff, M.; Hägglund, C. Atomic Layer Deposition of Cubic and Orthorhombic Phase Tin Monosulfide. Chem. Mater. 2017, DOI: 10.1021/acs.chemmater.6b05323.

(23) Ren, Y.; Ross, N.; Larsen, J. K.; Rudisch, K.; Scragg, J. J. S.; Platzer-Björkman, C. Evolution of $\mathrm{Cu}_{2} \mathrm{ZnSnS}_{4}$ during Non-Equilibrium Annealing with Quasi-in Situ Monitoring of Sulfur Partial Pressure. Chem. Mater. 2017, DOI: 10.1021/acs.chemmater.7b00671.
(24) Scragg, J. J.; Watjen, J. T.; Edoff, M.; Ericson, T.; Kubart, T.; Platzer-Bjorkman, C. A Detrimental Reaction at the Molybdenum Back Contact in $\mathrm{Cu}_{2} \mathrm{ZnSn}(\mathrm{S}, \mathrm{Se})_{4}$ Thin-Film Solar Cells. J. Am. Chem. Soc. 2012, 134, 19330-3. 